\title{
The Effect of Annealing Heat Treatment on Structural and Optical Properties of Ce-doped ZnO Thin Films
}

\author{
Shu Cui ${ }^{1,2, a}$, Chengyou Liü ${ }^{1, b}$, Xiaotian $\mathrm{Li}^{2, \mathrm{c}, \text { * }}$ \\ ${ }^{1}$ School of Physics, Tonghua Normal University, Tonghua, Jilin 134002, China \\ ${ }^{2}$ School of Material Science and Engineering, Key laboratory of Automobile Materials of Ministry of \\ Education, Jilin University, Changchun 130012, China \\ accuishu20132013@163.com, bchengyouliu2013@163.com, cxiaotianli@jlu.edu.cn
}

Keywords: Sol-gel method, Ce-doped $\mathrm{ZnO}$ thin films, annealing heat treatment, Photoluminescence.

\begin{abstract}
Ce-doped $\mathrm{ZnO}$ thin films were deposited on simple glass substrate and $\mathrm{SiO} 2$ substrate by sol-gel technique. The characteristics were studied by X-ray diffraction (XRD), atomic force microscope (AFM) and photoluminescence (PL) spectroscopy, respectively. The X-ray diffraction (XRD) experiments shows that most of the samples are highly c-axis oriented, furthermore, the c-axis oriented was improved as the annealing temperature arised. The PL spectrum shows obvious peak near 390nm. The intensity of eigen peak was improved as the annealing temperature arised, but the intensity of peaks in visible region was restrained as the annealing temperature arised. The morphology of the samples studied by the AFM shows an increase in the annealing temperature causes the surface flatter and the grain size larger.
\end{abstract}

\section{Introduction}

$\mathrm{ZnO}$ is a promising optoelectronic semiconductor material with a wide band gap of $3.37 \mathrm{eV}$ at room temperature, large exciton binding energy of $60 \mathrm{meV}$. $\mathrm{ZnO}$ is considered to be one of the most promising materials with the advantages of abundance in nature, excellent chemical and thermal stability, and its specific electrical and optoelectronic property. Furthermore, the excellent properties of the high exciton binding energy and the large internal piezoelectric coefficient, making ZnO thin films useful in solar cells, light emitting diodes, gas sensing devices and transistors $[1,2]$.

Preparation and properties study of doped and undoped $\mathrm{ZnO}$ thin films are currently research focus. In particular, great attention has been paid on the study of ZnO-based diluted magnetic semiconductors(DMS), however, In contrast with numerous experiments were performed to study ferro magnetic element doped $\mathrm{ZnO}$ materials, little study was done on the RE-doped $\mathrm{ZnO}$ materials, there are still challenges in this area, such as there is no agreement about magnetic source, the unsatisfied repeatability to get uniform, stable products of RE-doped ZnO-based DMS.

So far, many deposition techniques have been developed to prepare ZnO-based thin films, such as pulsed laser deposition (PLD) [3], magnetron sputtering [4], molecular beam epitaxy (MBE) [5], Metal organic chemical vapor deposition (MOCVD) [6], sol-gel method [7], etc. Compared with other preparation methods, sol-gel method has more cost advantages with simple equipment, process easy to control, high product purity and uniformity, so this method has promising application for industrial growth of large area.

In this paper, we have chosen Ce as a dopant [8.9], in view of the similar facet space of $\mathrm{ZnO}$ and $\mathrm{CeO}$. We successful prepared Ce-doped $\mathrm{ZnO}$ thin films on ordinary glass and $\mathrm{SiO}_{2}$ substrates using the sol-gel method, furthermore, we studied the influence of annealing temperature on the properties of structure, morphology and luminescence. 


\section{Preparation and Characterization}

\subsection{Synthesis of $\mathrm{ZnO}$ thin films.}

The zinc acetate dehydrate was used as starting material, the mono-ethanolamine (MEA) was used as stabilizing agent, and the ethylene glycol served as solvent. All reagents are of analytical grade without further purification. Firstly, a certain amount of hydrated zinc acetate dissolved in a certain amount of ethylene glycol, then, the mono-ethanolamine in equal mole ratio as zinc acetate dehydrate was added, furthermore the mixed solution was stirred in a high pressure reactive kettle for 1 hour at $60^{\circ} \mathrm{C}$ to yield a clear and homogeneous solution. The solution was aged at room temperature for 24 hours. Secondly, the $\mathrm{ZnO}$ thin films deposited on simple glass were carried out at a spinning speed of $3000 \mathrm{rpm}$ for $10 \mathrm{~s}$. The samples then were dried at $200^{\circ} \mathrm{C}$ for $10 \mathrm{~min}$. The procedure was continuously repeated 10 times at the same substrate to increase the films thickness. Finally, the films were annealed in air at $500^{\circ} \mathrm{C}$ for 1 hour, we labeled it as a.

\subsection{Sythesis of Ce-doped $\mathrm{ZnO}$ thin films.}

To get the production of Ce-doped $\mathrm{ZnO}$ thin films, cerium nitrate hexahydrate was added to the previously mentione, the molar ratio of Ce to $\mathrm{Zn}$ was $1 \%$. The mixed clear and homogeneous solution was aged at room temperature for 24 hours, then, the same spining procedure was carried out. Finally, the films were annealed in air at $950^{\circ} \mathrm{C}, 1000^{\circ} \mathrm{C}, 1100^{\circ} \mathrm{C}$ for 1 hour, and we labeled the samples as b, c, d.

\subsection{Characterizations}

The structure of the Ce-doped $\mathrm{ZnO}$ thin films were characterized by the X-ray diffraction(XRD) which pattern was measured on a D/max-RA XRD spectrometer with a $\mathrm{CuK} \alpha$ line of $0.15406 \mathrm{~nm}$. The morphology was studied by atom force microscope (AFM). The band gap of the films were investigated by Transmission spectra and photoluminescence (PL), and the spectrophotometer was excited by a continuous He-Cd laser at a wavelength of 325nm.

\section{Results and Discussion}

The phase and purity of the as-prepared samples were examined by XRD shown in Fig.1. All of the thin films display sharp peaks corresponding to the hexagonal wurtzite structure. The intensities of these peaks increased and the full width at half-maximum narrow down as the annealing temperature improving from $500^{\circ} \mathrm{C}$ to $1100^{\circ} \mathrm{C}$, the sample annealed at $1000^{\circ} \mathrm{C}$ presents the best structure property because of no diffraction peaks of other impurity phases were found in the samples, this means that the Ce ions had uniformly replaced the $\mathrm{Zn}^{2+}$ or entered into interstitial sites in the $\mathrm{ZnO}$ lattice. However, there appeared lots of impurity phases of the sample annealed at $1100^{\circ} \mathrm{C}$, suggesting that only part of dopant added to the $\mathrm{ZnO}$ lattice. The XRD results tell that the crystalline characteristic of the sample has improved with the increasing of annealing temperature to a certain extent. 


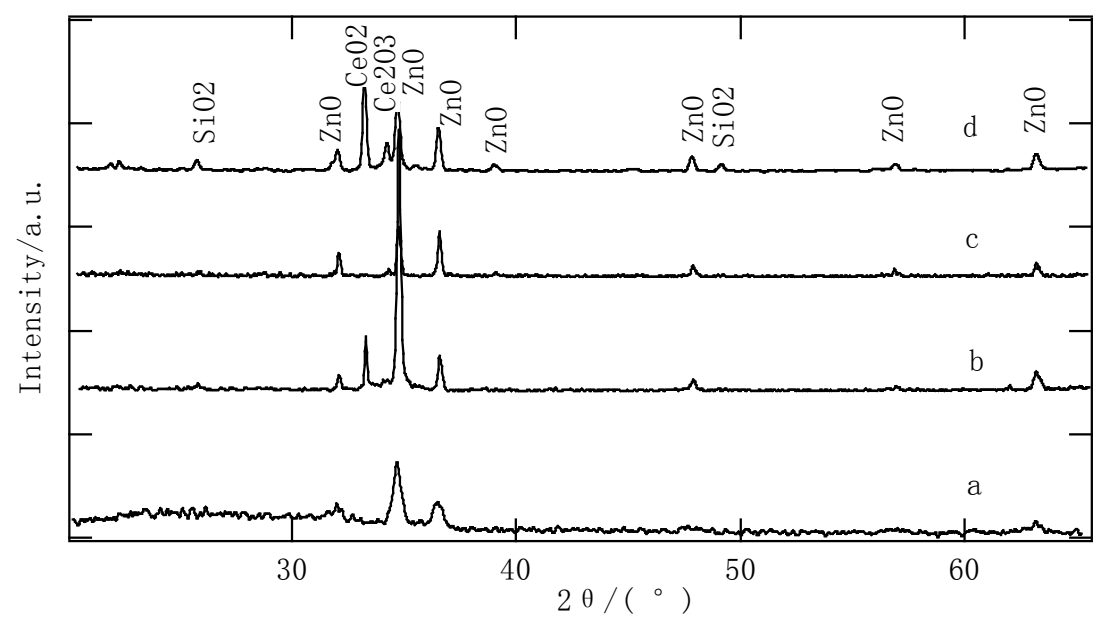

Fig.1 X-ray diffraction pattern of Ce doped $\mathrm{ZnO}$ thin films.

Figure.2. shows the morphology informations of the samples annealed at different temperature. Generally speaking, all of the samples have smooth surface. The obvious nano-cluster we can see in Figure $2 \mathrm{~b}$ to $2 \mathrm{~d}$, then, the grain size became larger as the annealing temperature increasing, which is consistent with fig.1, yet, the sample $d$ in the figure 2 shows obvious noisy signal, suggesting that the flatness of the sample come down at the annealing temperature of $1100^{\circ} \mathrm{C}$, this once again proved that the annealing temperature plays a big role on the sample surface morphology, different annealing temperature can get completely different sample surface morphology.

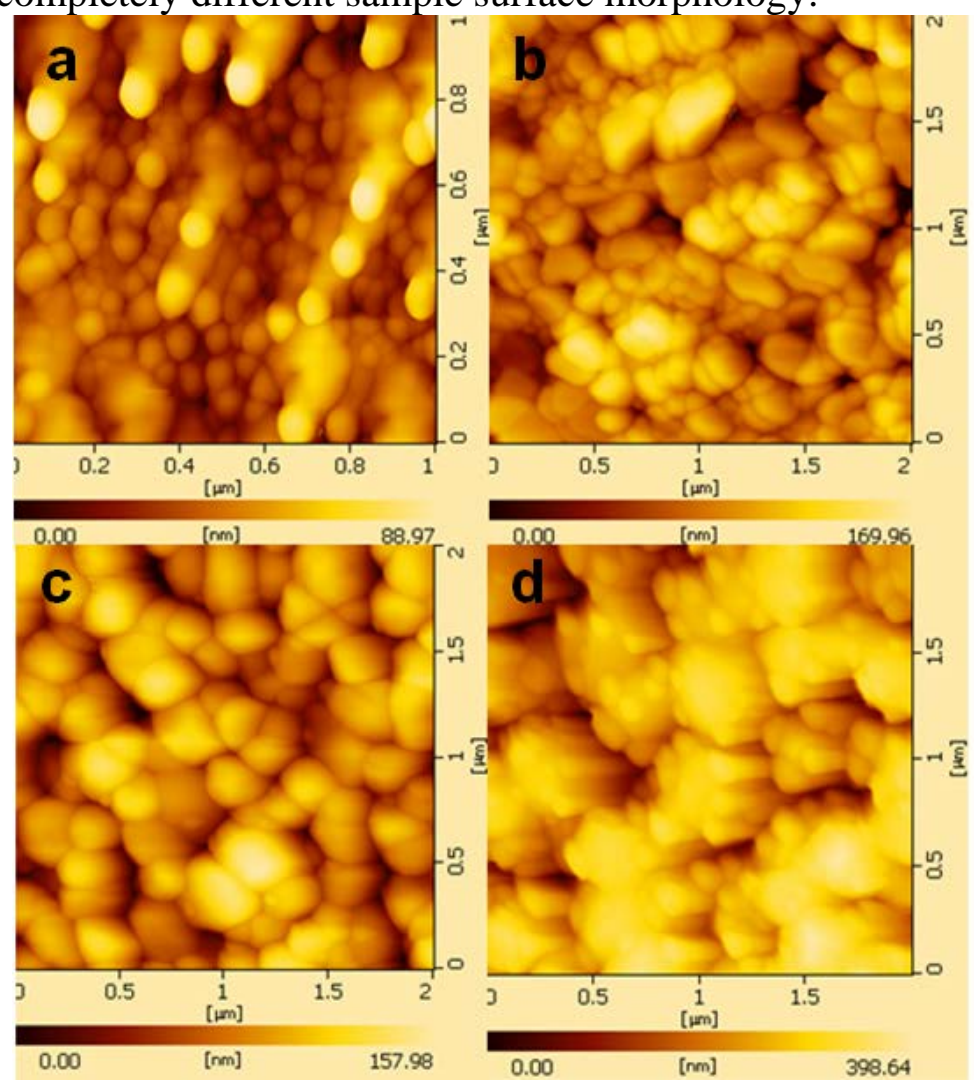

Fig.2. AFM images of Ce-doped $\mathrm{ZnO}$ thin films annealed at different temperature. 


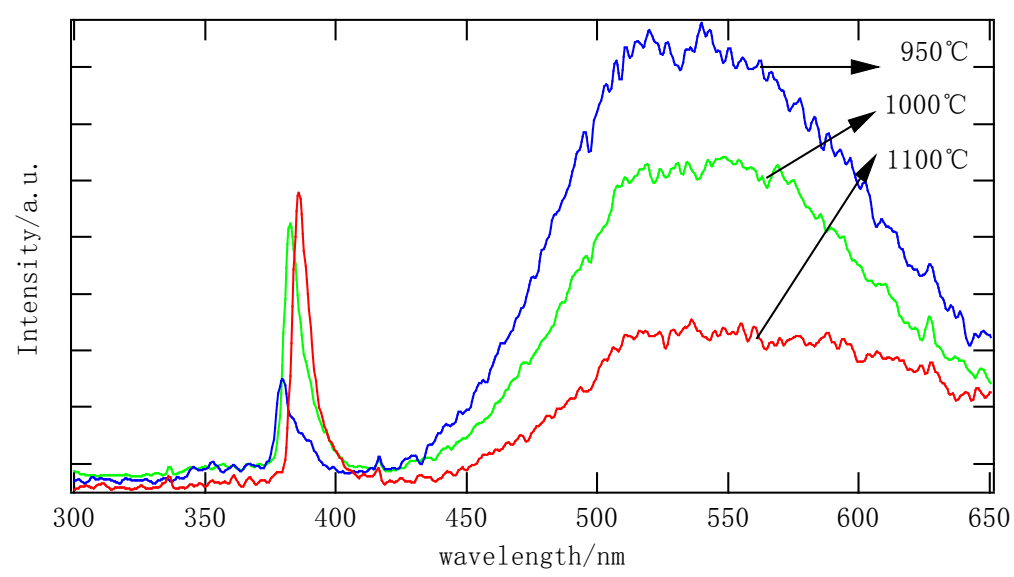

Fig.3. Photoluminescence spectra of Ce-doped $\mathrm{ZnO}$ thin films.

In order to study the photoluminescence properties of as-prepared Ce-doped $\mathrm{ZnO}$ thin films, we measured the PL spectra of $\mathrm{ZnO}$ thin films, it can be seen from Fig.3. All of the samples annealed at $950^{\circ} \mathrm{C}, 1000^{\circ} \mathrm{C}, 1100{ }^{\circ} \mathrm{C}$ contain two emission bands. One was the near band edge emission in the UV region, as we know [10], it was attributed to free-exciton recombination, and the other was the visible light emission, which was produced by the transition of the excited optical center, from the deep level to the valence level, and such a deep level emission was usually caused by the structural defect and impurities. Furthermore, the positions and intensities of the emission bands were both affected by annealing temperature. The affection shows in three aspects. Firstly, the intensity of the violet peak is enhanced, which means annealing temperatures can be helpfully improve sample intrinsic luminescence properties. Secondly, the position shows an obvious redshift which means the band-gap energy get smaller, it is generally believed this is due to the lattice distortion. Finally, the emission band of $450 \mathrm{~nm}$ to $450 \mathrm{~nm}$ shows a certain restrain with the annealing temperature increasing, this may be due to rare earth $\mathrm{Ce}$ is hard to mix with $\mathrm{ZnO}$ lattice structure at the high temperature.

\section{Summary}

We successfully prepared Ce-doped $\mathrm{ZnO}$ thin films on glasses substrates and $\mathrm{SiO} 2$ substrates by sol-gel method. In this paper, we mainly studied the effect of annealing temperature on the Ce-doped $\mathrm{ZnO}$ thin film. The sample annealed at $1000^{\circ} \mathrm{C}$ has the best comprehensive performance, but in the XRD spectrum of the sample annealed at $1100^{\circ} \mathrm{C}$ there are lots of impurity phases, and the surface roughness of this sample decreased obviously. General speaking, the result of structure, morphology and optical properties demonstrates that the comprehensive property of Ce-doped ZnO thin films prepared by sol-gel method can improved as the annealing temperature increasing to a certain extent.

\section{Acknowledgments}

This word was supported by the National Natural Science Foundation of China for Project Nos.21076094 and the Program for the development of Science and Technology of Jilin province (Item No. 200705C01).

\section{References}

[1] S.J.Pearton, D.P. Norton, K. Ip, Y.W. Heo and T. Steiner. Prog. Mater. Sci., Vol. 50 (2005) No.3, p.293.

[2] Z.K.Tang , G.K.L. Wong and P.Yu . Appl. Phys.Lett., Vol. 72 (1998) No.25, p.3270.

[3] M. Sugiura, Y. Nakashima, et al. Applied surface science, 197-198(2002), 472-474. 
[4] W. Lin, R. Ma, et al. Applied surface science, 253(2007), 5179-5183.

[5] N.Y. Yuan, L.F, J.H. Li and X.Q. Wang. Applied Surface Science, Vol. 253 (2007) No.11, p. 4990.

[6] J. H. Yang, M. Gao, etal. CHEM. RES. CHINESE UNIVERSITIES. 2008, 24(3), 266-269.

[7] K.M. Lin and P. Tsai. Thin Solid Films, Vol. 515(2007) No.24, p. 8601.

[8] B. Cheng, Y. Xiao, G. Wu, et al. Appl. Phys. Lett., 2004, 84, 416.

[9] Z. Bofiani, B. Derkowska, P. Dalasinski, et al. Optics Communications, 2006, 267, 433.

[10]K. Vanheusden, W. L. Warren, C. H. Seager, et al. J. Appl. Phys.,1996, 79, 7983. 